\title{
Performance of bioprostheses and mechanical prostheses assessed by composites of valve-related complications to 15 years after mitral valve replacement
}

W. R. E. Jamieson, MD, O. von Lipinski, MD, R. T. Miyagishima, MD, L. H. Burr, MD, M. T. Janusz, MD, H. Ling, MD, G. J. Fradet, MD, F. Chan, and E. Germann, MSc

From the University of British Columbia, Vancouver, British Columbia, Canada.

Read at the Twenty-ninth Annual Meeting of The Western Thoracic Surgical Association, Carlsbad, Calif, June 18-21, 2003.

Received for publication June 17, 2003; revisions received Sept 8, 2004; accepted for publication Sept 22, 2004.

Address for reprints: W. R. Eric Jamieson, MD, 486 Burrard Building, St Paul's Hospital, 1081 Burrard St, Vancouver, BC V6Z 1Y6, Canada (E-mail: wrej@ interchange.ubc.ca).

J Thorac Cardiovasc Surg 2005;129:1301-8

$0022-5223 / \$ 30.00$

Copyright $\odot 2005$ by The American Association for Thoracic Surgery

doi:10.1016/j.jtcvs.2004.09.042
Objective: Predominant concerns of patients undergoing valve replacement surgery are risks of death, stroke, antithrombotic bleeding, and reoperation related to the replacement prosthesis. The purpose of this study was to compare valve-related reoperation, morbidity (permanent impairment), and mortality between bioprostheses and mechanical prostheses for mitral valve replacement.

Methods: Between 1982 and 1998, a total of 959 bioprostheses were implanted in 943 patients, and a total of 961 mechanical prostheses were implanted in 839 patients. Total follow-ups were 5730 years for bioprostheses and 5271 years for mechanical prostheses. Eight variables were considered as predictors of risk for the composites of valve-related complications.

Results: The linearized occurrence rates for valve-related reoperation were 3.7 events/100 patient-years for bioprostheses and 0.5 events $/ 100$ patient-years for mechanical prostheses $(P<.001)$, with all age groups differentiated except older than 70 years. Valve-related morbidity was undifferentiated for bioprostheses and mechanical prostheses. Valve-related mortalities were 1.7 events/100 patient-years for bioprostheses and 0.7 events $/ 100$ patient-years for mechanical prostheses $(P<$ $.001)$. Predictors of valve-related reoperation were age and valve type. The only predictor of valve-related morbidity was age, whereas age and valve type were predictors for valve-related mortality. Actual freedom from valve-related reoperation favored mechanical prostheses in all age groups except older than 70 years $(91.7 \% \pm 2.0 \%$ for bioprostheses at 15 years and $96.7 \% \pm 1.5 \%$ at 12 years for mechanical prostheses). Actual freedom from valve-related morbidity was not different between bioprostheses and mechanical prostheses. Actual freedom from valve-related mortality favored mechanical prostheses in all groups except older than 70 years.

Conclusion: Comparative evaluation gives high priority in mitral valve replacement for mechanical prostheses relative to bioprostheses for freedom from valve-related reoperation and valve-related mortality but not valve-related morbidity. Freedom from valve-related reoperation and valve-related mortality favors mechanical prostheses for all age groups except older than 70 years. Valve-related morbidity, due to neurologic or functional impairments, does not differentiate between bioprostheses and mechanical prostheses.

$\mathrm{T}$ he long-term performance of bioprostheses and mechanical prostheses in aortic and mitral valve replacement has only been compared in two large randomized trials. ${ }^{1-5}$ These trials assessed patient survival and valve-related complications. There have been no comparisons of long-term performance of bioprostheses and mechanical prostheses considering composites of valve-related complications with actuarial and actual methodologies. Jamieson and colleagues ${ }^{6}$ 
used the actual methodology in 2000 in reporting the performance of porcine bioprostheses. The actual freedoms from valve-related mortality at 15 years were $85 \%$ for aortic valve replacement and $80 \%$ for mitral valve replacement in patients older than 60 years. The actual freedoms from valve-related reoperation at 15 years were $88 \%$ in patients aged 61 to 70 years and $96 \%$ in those older than 70 years for aortic valve replacement and $93 \%$ in patients older than 70 years for mitral valve replacement. The purpose of this study was to evaluate composites of valve-related complications (valve-related mortality, morbidity with permanent impairment, and reoperation) in populations receiving bioprostheses and mechanical prostheses. The patients' primary concerns are these long-term risks.

\section{Methods}

Between 1982 and 1998, a total of 959 bioprostheses were implanted in 943 patients, and a total of 961 mechanical prostheses were implanted in 839 patients. The patients considered for the study had undergone no previous cardiac procedures. The overall mean age differed by prosthesis type: $65.4 \pm 11.7$ years for bioprostheses and $59.1 \pm 11.8$ years for mechanical prostheses $(P$ $<.001)$ but age category breakdown $(<40,41-50,51-60,61-70$, and $>70$ years) did not. Concomitant coronary artery bypass grafting $(\mathrm{CABG})$ was performed at the initial procedure or valverelated reoperation for $42.9 \%$ of bioprostheses $(n=411)$ or $31.1 \%$ of mechanical prostheses $(\mathrm{n}=299, P<.001)$. The total follow-up differed by prosthesis group: 5730.3 years for bioprostheses and 5271.3 years for mechanical prostheses $(P=.002)$. The follow-up also differed by prosthesis group for the age categories. Completeness of follow-up figures were $99.0 \%$ for bioprostheses and $95.4 \%$ for mechanical prostheses.

During the 17-year implantation period, the bioprosthesis types and numbers of implants were as follows: Carpentier-Edwards SAV (Edwards Lifesciences, Irvine, Calif; $\mathrm{n}=857$ ), CarpentierEdwards PERIMOUNT (Edwards Lifesciences; $\mathrm{n}=33$ ), and Medtronic Mosaic (Medtronic, Inc, Minneapolis, Minn; $n=53$ ), all second and third generation porcine bioprostheses. During this period the mechanical prostheses were as follows: St Jude Medical (St Jude Medical Inc, Minneapolis, Minn; $\mathrm{n}=471$ ) and CarboMedics (Sorin CarboMedics Inc, Austin, Tex, $\mathrm{n}=313$ ). The overall valve sizes at the initial procedure were $28.4 \pm 2.2 \mathrm{~mm}$ for bioprostheses and $28.2 \pm 2.3 \mathrm{~mm}$ for mechanical prostheses $(P=$ $.042)$.

\section{Follow-up Procedure}

The patients were followed up periodically throughout the observation period, with the closing interval in the year 2000 to 2001. The patients were evaluated by telephone interviews, and health records and consultations were evaluated when appropriate. The documentation on reoperative procedures was fully reviewed. The vital statistics death registry was used to ensure completeness of mortality data.

\section{Study End Points}

The three primary end points were valve-related mortality (inclusive of reoperative mortality), valve-related morbidity, and valve- related reoperation, as defined by "Guidelines for Reporting Morbidity and Mortality After Cardiac Valvular Operations" from the The Society of Thoracic Surgeons, The American Association for Thoracic Surgery, and European Association for Cardio-Thoracic Surgeons. ${ }^{7}$ Valve-related mortality included death caused by structural valve deterioration, nonstructural dysfunction, thrombosis, thromboembolism, hemorrhage, or prosthetic valve endocarditis and death related to reoperation for a valve-related complication. Valve-related mortality was inclusive of sudden unexplained, unexpected deaths. Valve-related reoperation was reoperation for any valve-related complication. Valve-related morbidity was considered, for this study, as permanent valve-related impairment as a result of permanent neurologic or other functional deficit caused by structural valve deterioration, nonstructural valve dysfunction, valve thrombosis, thrombotic embolism, bleeding, prosthetic valve endocarditis, or reoperation.

All valve-related complications were used to calculate the composites of valve-related mortality, valve-related reoperation, and valve-related morbidity, with these composites being attributed to the particular valve type (either bioprosthesis or mechanical prosthesis). When patients had a bioprosthesis explanted and a mechanical prosthesis implanted or vice versa, they were censored from the former group and entered into the latter group. Operative mortality (30-day mortality) was attributed to the explanted prosthesis and classified as valve-related mortality. It is for this reason the study did not evaluate patient survival, because a portion of the patients were in both the bioprosthesis and mechanical prosthesis arms of the study.

The variables considered as predictors of risk for the composites of valve-related complications were prosthesis type, age (continuous variable), age group (categoric variable), sex, prosthesis size, and concomitant CABG. All patients undergoing initial cardiac operations between 1982 and 1998, as well as reoperation in 1999,2000 , or 2001, were included in the analysis.

\section{Statistical Analysis}

Differences in baseline characteristics between the two groups were compared with the $t$ test for continuous variables and $\chi^{2}$ for categoric variables; no adjustment for multiple comparisons was made. The composites of valve-related complications were calculated for freedom from the complications composite with the Kaplan-Meier actuarial methodology and compared by the logrank statistic. The actual freedom or cumulative incidence was determined by the modified Kaplan-Meier methodology of Grunkemeier and colleagues. ${ }^{8-11}$ The composites of complications were also evaluated as linearized occurrence rates as events/100 patientyears, with comparisons made with the log likelihood ratio statistic. Linearized occurrence rates are not recommended for evaluation of structural valve deterioration reoperation because of lack of constant hazard with time but an advancing hazard with time. The Weibull distribution curve is the appropriate representation of structural valve deterioration and structural valve deterioration reoperation. Linearized occurrence rates were used in this study to depict valve-related reoperation, because valve-related reoperation was inclusive of all causes of valve-related reoperation: prosthetic valve endocarditis, nonstructural dysfunction (periprosthetic leak), and prosthesis thrombosis, as well as structural valve deterioration. 
TABLE 1. Linearized occurrence rates of composites of valve-related complications by age group

\begin{tabular}{|c|c|c|c|c|c|c|c|c|c|}
\hline \multirow[b]{2}{*}{$\begin{array}{l}\text { Age } \\
\text { (y) }\end{array}$} & \multicolumn{3}{|c|}{ Reoperation } & \multicolumn{3}{|c|}{ Morbidity } & \multicolumn{3}{|c|}{ Mortality } \\
\hline & BP & MP & $\begin{array}{c}P \\
\text { value }\end{array}$ & BP & MP & $\begin{array}{c}P \\
\text { value }\end{array}$ & BP & MP & $\begin{array}{c}P \\
\text { value }\end{array}$ \\
\hline$\leq 40$ & 7.1 & 0.7 & $<.001$ & 0.7 & 1.8 & .1715 & 0.7 & 0.9 & .8088 \\
\hline $41-50$ & 6.4 & 0.4 & $<.001$ & 0.7 & 1.8 & .0812 & 0.9 & 0.2 & .0905 \\
\hline $51-60$ & 5.3 & 0.5 & $<.001$ & 1.3 & 1.2 & .7667 & 1.0 & 0.3 & .0125 \\
\hline $61-70$ & 3.3 & 0.5 & $<.001$ & 1.6 & 1.9 & .5318 & 2.2 & 0.8 & .0002 \\
\hline$>70$ & 1.2 & 0.6 & 1907 & 2.1 & 2.8 & .8954 & 2.1 & 1.9 & .7194 \\
\hline Total & 3.7 & 0.5 & $<.001$ & 1.5 & 1.7 & .3140 & 1.7 & 0.7 & $<.001$ \\
\hline
\end{tabular}

$B P$, Bioprostheses; $M P$, mechanical prostheses.

The predictors of the complications composites were determined by univariate analysis and multiple Cox hazard regression analysis.

\section{Results}

The linearized occurrence rates for the composites of complications are detailed in Table 1. The linearized rate for overall valve-related reoperation was 3.7 events/100 patient-years for bioprostheses and 0.5 events/100 patientyears for mechanical prostheses $(P<.001)$. The rates were also different overall for valve-related mortality, at 1.7 events/100 patient-years for bioprostheses and 0.7 events/ 100 patient-years for mechanical prostheses $(P<.001)$. The rates were not different ( $P$ not significant) for valve-related morbidity, at 1.5 events/100 patient-years for bioprostheses and 1.7 events/100 patient-years for mechanical prostheses.

Valve-related reoperation favored mechanical prostheses for all age groups except older than 70 years, for which the bioprosthesis rate was 1.2 events/100 patient-years and the mechanical prosthesis rate was 0.6 events/100 patient-years $(P=.1907)$. Valve-related mortality only favored mechanical prostheses relative to bioprostheses for the age groups 51 through 60 years and 61 through 70 years. For patients older than 70 years, the rates were similar, at 2.1 events $/ 100$ patient-years for bioprostheses and 1.9 events/100 patientyears for mechanical prostheses $(P=.7194)$. Valve-related morbidity was undifferentiated between mechanical prostheses and bioprostheses for all age groups.
The predictors of valve-related composites of complications are presented in Table 2. The predictors of valverelated reoperation were age and prosthesis type. The mean age at implantation of patients who underwent a reoperation $(\mathrm{n}=240)$ was $56.0 \pm 12.9$ years; that for those who did not $(\mathrm{n}=1680)$ was $63.4 \pm 11.7$ years $(P<.001)$. The hazard ratio (HR) was 0.98 (95\% confidence interval 0.97-0.99, $P=.0001)$. Reoperation for prosthesis type was performed in $2.7 \%$ of mechanical prosthesis cases $(n=26)$ and $22.2 \%$ of bioprosthesis cases $(\mathrm{n}=214, P=.001)$. The HR of valve type for valve-related reoperation was 0.19 (95\% confidence interval $0.13-0.29, P<.001$, bioprostheses greater than mechanical prostheses). Among those who underwent valve-related reoperation, concomitant CABG was performed at initial surgery or valve-related reoperation in 8.6\% (n = 61/710) and not in 14.8\% $(\mathrm{n}=179 / 1210, P<$ $.0001)$. The HR for concomitant $\mathrm{CABG}$ was $1.2(95 \%$ confidence interval $0.9-1.7, P=.1278$ ).

Age was the only predictor of valve-related morbidity, with HR 1.02 (95\% confidence interval 1.001-1.031, $P=$ $.0302)$. Age and valve type were the predictors of valverelated mortality. The mean age of patients with valverelated mortality $(\mathrm{n}=133)$ was $65.3 \pm 10.6$ years, and that of patients who did not $(\mathrm{n}=1787)$ was $62.2 \pm 12.2$ years $(P<.002)$. The HR for age as a continuous variable was 1.041 (95\% confidence interval 1.02-1.06, $P<.001$ ). The HR for valve type was 0.61 (95\% confidence interval $0.41-$ $0.93, P=.0202$. Valve-related mortality occurred in $8.5 \%$ of female patients $(\mathrm{n}=88 / 1034)$ and $5.1 \%$ of male patients (n $=45 / 886, P=.00421)$. The HR was 1.4 (95\% confidence interval 0.9-2.1, $P=.0912$, female patients greater than male patients). Prosthesis type was predictive (bioprostheses $10.1 \%$ [ $\mathrm{n}=97 / 963]$, mechanical prostheses $3.8 \%$ $[\mathrm{n}=36 / 957])$ for valve-related mortality $(P<.001$, or 1.7 events/100 patient-years for bioprostheses and 0.7 events/ 100 patient-years for mechanical prostheses).

The freedom ( \pm SD) from composites of valve-related complications are detailed for actual and actuarial freedom to 15 years from valve-related reoperation, valve-related morbidity, and valve-related mortality in Figures 1 through 6 for age groups 61 through 70 and older than 70 years.

TABLE 2. Predictive risk factors

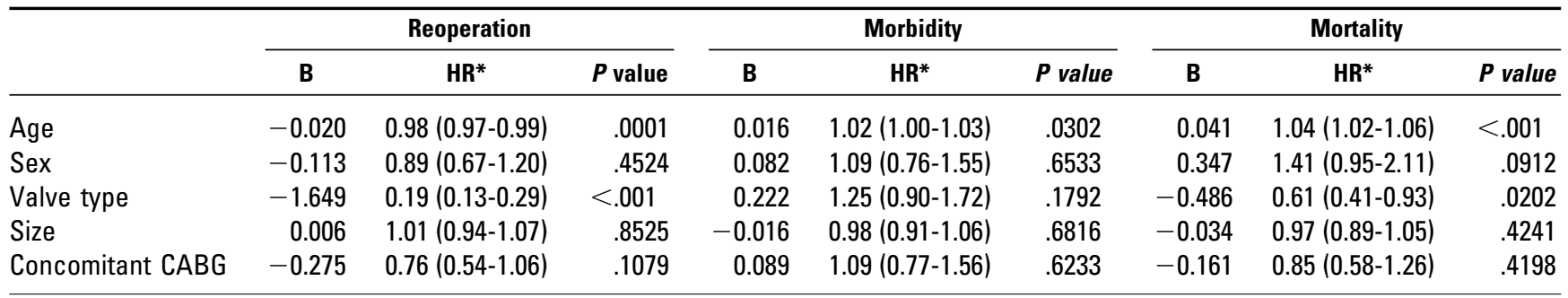

Baseline assumptions for dichotomous variables: Sex, male; valve type, bioprosthesis; concomitant CABG; $B$, Regression coefficient. *The $95 \%$ confidence interval for the hazard ratio is given in parentheses. 


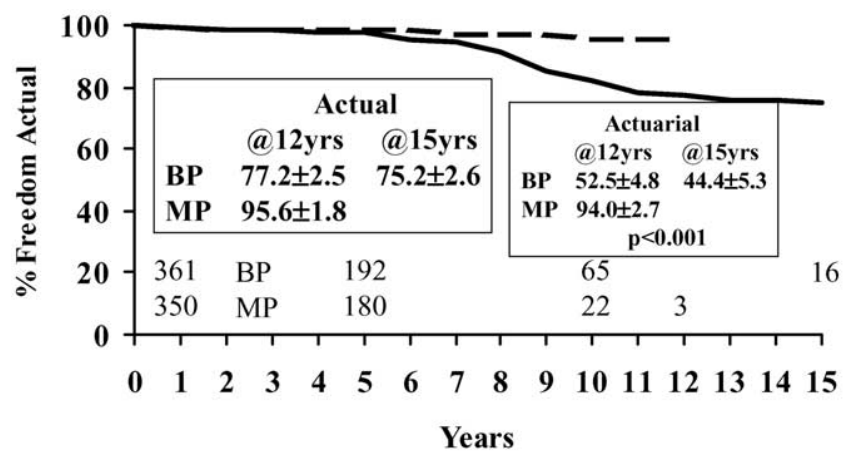

Figure 1. Freedom from valve-related reoperation (actual); 61 through $\mathbf{7 0}$ years. Solid line, Bioprostheses; dashed line, mechanical prostheses.

The actuarial freedom from valve-related reoperation at 15 years was significantly greater for mechanical prostheses than for bioprostheses for all age groups other than older than 70 years $(P<.05)$. The actuarial freedoms for the group older than 70 years were $82.3 \% \pm 4.8 \%$ for bioprostheses and $96.7 \% \pm 1.6 \%$ at 11 years for mechanical prostheses ( $P$ not significant). The actual freedom from valve-related reoperation trended higher for mechanical prostheses than for bioprostheses for all age groups except older than 70 years. For the group older than 70 years, the actual freedoms from valve-related reoperation were $91.7 \%$ $\pm 2.0 \%$ for bioprostheses at 15 years and $96.7 \% \pm 1.5 \%$ for mechanical prostheses at 11 years.

The actuarial freedom from valve-related morbidity for all age groups was undifferentiated between bioprostheses and mechanical prostheses ( $P$ not significant). The actual freedom from valve-related morbidity was also undifferentiated for all age groups between bioprostheses and mechanical prostheses.

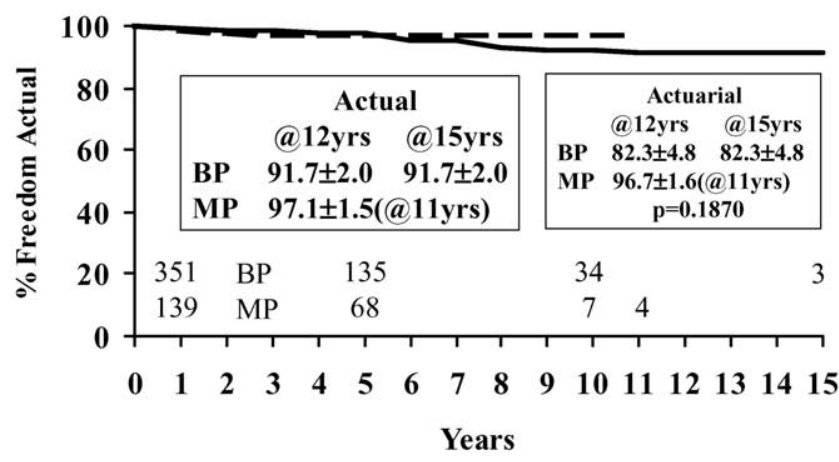

Figure 2. Freedom from valve-related reoperation (actual); older than $\mathbf{7 0}$ years. Solid line, Bioprostheses; dashed line, mechanical prostheses.

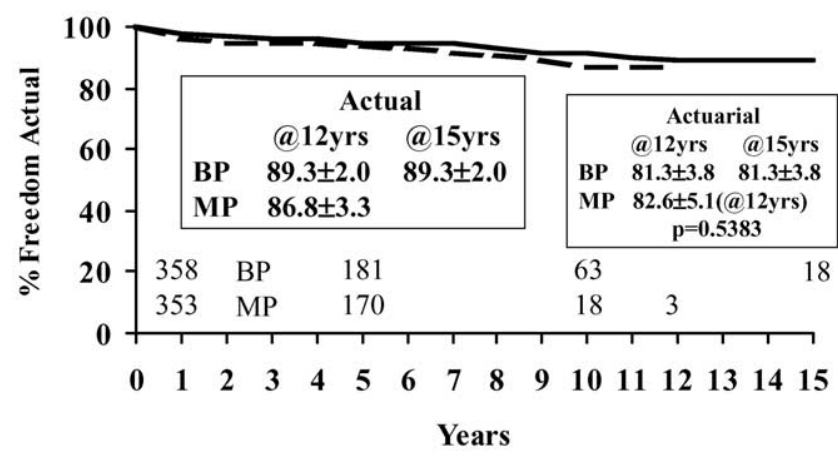

Figure 3. Freedom from valve-related morbidity (actual); 61 through 70 years. Solid line, Bioprostheses; dashed line, mechanical prostheses.

The actuarial freedom from valve-related mortality was only different for age 51 through 60 years, at $75.4 \% \pm 8.3 \%$ for bioprostheses and $87.5 \% \pm 8.7 \%$ for mechanical prostheses. There were identifiable trends in actual freedom in favor of mechanical prostheses relative to bioprostheses for age groups 41 through 50, 51 through 60, 61 through 70 , and older than 70 years.

The actual and actuarial freedoms from each of the composites for age groups 61 through 70 years and older than 70 years are demonstrated in Figures 1 through 6 . The actual freedoms from valve-related reoperation at 15 years for the age group 61 through 70 years were $75.2 \% \pm 2.6 \%$ for bioprostheses and $95.6 \% \pm 1.8 \%$ at 12 years for mechanical prostheses (Figure 1). The actual freedoms from valve-related reoperation for the age group older than 70 years were $91.7 \% \pm$ $2.0 \%$ for bioprostheses at 15 years and $97.1 \% \pm 1.5 \%$ at 11 years for mechanical prostheses (Figure 2).

The actual freedoms from valve-related morbidity for the age group 61-70 years were $89.3 \% \pm 2.0 \%$ for bioprostheses at 15 years and $86.8 \% \pm 3.3 \%$ at 12 years for mechan-

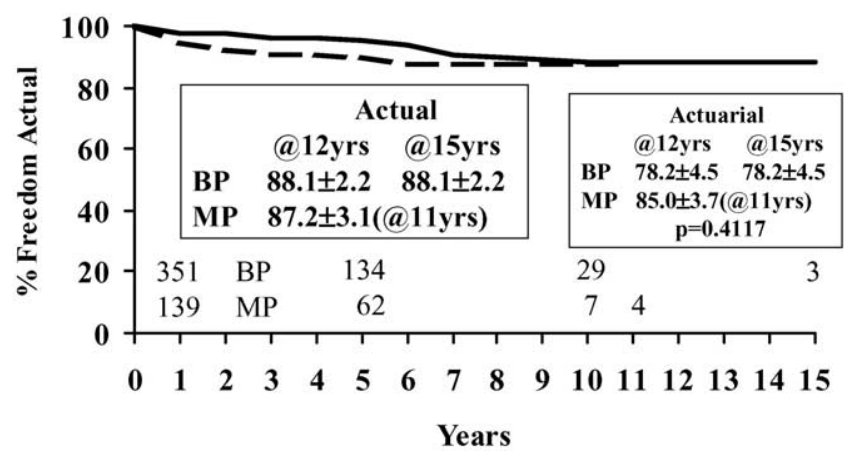

Figure 4. Freedom from valve-related morbidity (actual); older than $\mathbf{7 0}$ years. Solid line, Bioprostheses; dashed line, mechanical prostheses. 


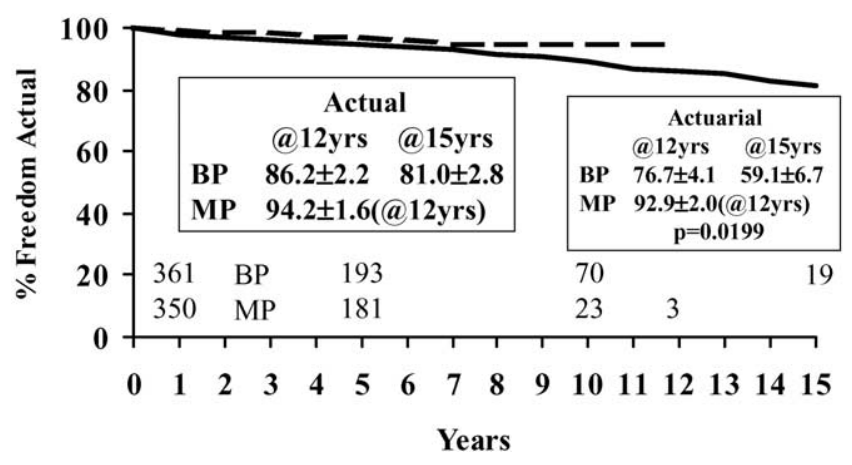

Figure 5. Freedom from valve-related mortality (actual); 61 through 70 years. Solid line, Bioprostheses; dashed line, mechanical prostheses.

ical prostheses (Figure 3). For the age group older than 70 years, the freedoms were similar at 15 and 12 years, $88.1 \% \pm$ $2.2 \%$ for bioprostheses and $87.2 \% \pm 3.1 \%$ at 11 years for mechanical prostheses, respectively (Figure 4).

The actual freedoms from valve-related mortality (favored mechanical prostheses relative to bioprostheses) for the age group 61 through 70 years were $81.0 \% \pm 2.8 \%$ for bioprostheses at 15 years and $94.2 \% \pm 1.6 \%$ for mechanical prostheses at 12 years (Figure 5). The actual freedom at 15 years from valve-related mortality for those older than 70 years was $84.3 \% \pm 2.8 \%$ for bioprostheses and at 11 years $90.7 \%$ $\pm 2.7 \%$ for mechanical prostheses.

There were 255 major thromboembolic events, with and without residual morbidity: 2.3 events/100 patient-years $(\mathrm{n}=129)$ for bioprostheses and 2.4 events/100 patientyears $(n=126)$ for mechanical prostheses. There were 118 bleeding events with and without residual morbidity: 0.5 events/100 patient-years $(n=27)$ for bioprostheses and 1.1 events/100 patient-years $(n=57)$ for mechanical prostheses. Of these major events, 178 were associated with residual morbidity. For thromboembolism $(\mathrm{n}=94)$, the rate for bioprostheses was 1.0 events/100 patient-years $(n=59)$, and that for mechanical prostheses was 0.7 events/100 patient-years $(n=35)$. For bleeding $(n=84)$, the rate for bioprostheses was 0.5 events/100 patient-years $(n=27)$, and that for mechanical prostheses was 1.1 events/100 patient-years $(\mathrm{n}=57)$. Of these 178 residual morbidity events, 161 were initial events: 88 thromboembolic events (55 for bioprostheses and 33 for mechanical prostheses) and 73 bleeding events (23 for bioprostheses and 50 for mechanical prostheses).

There were 214 reoperations in the bioprosthesis group and 26 in the mechanical prosthesis group. The distribution by cause of first events was nonstructural dysfunction for 15 bioprostheses and 12 mechanical prostheses, prosthetic valve endocarditis for 14 bioprostheses and 2 mechanical

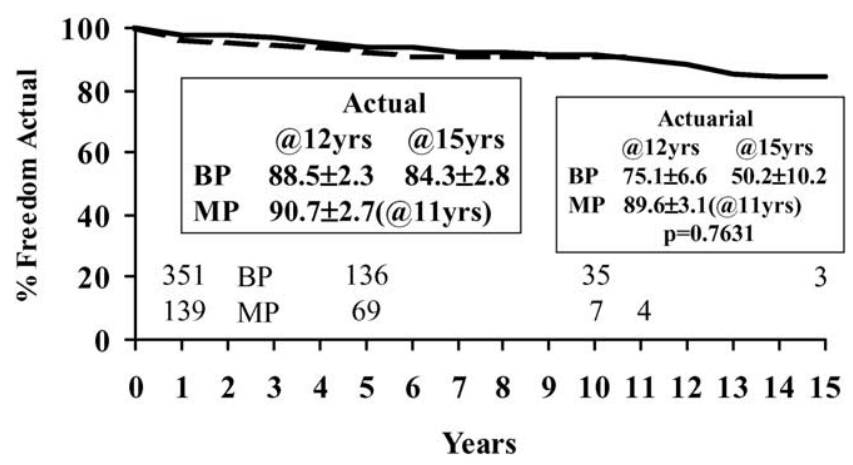

Figure 6. Freedom from valve-related mortality (actual); older than $\mathbf{7 0}$ years. Solid line, Bioprostheses; dashed line, mechanical prostheses.

prostheses, structural valve deterioration for 180 bioprostheses $(\mathrm{n}=53$ for $51-60$ years, $\mathrm{n}=62$ for $61-70$ years, $\mathrm{n}=$ 11 for $>70$ years) and 0 mechanical prostheses, and thrombosis for 5 bioprostheses and 12 mechanical prostheses. There were 97 valve-related deaths in the bioprosthesis group and 36 in the mechanical prosthesis group. The distribution by cause of first events was bleeding for 9 bioprostheses and 14 mechanical prostheses, nonstructural dysfunction for 7 bioprostheses and 2 mechanical prostheses, prosthetic valve endocarditis for 9 bioprostheses and 4 mechanical prostheses, structural valve deterioration for 34 bioprostheses ( $\mathrm{n}=20$ for $61-70$ years, $\mathrm{n}=9$ for $>70$ years) and 0 mechanical prostheses, thromboembolism for 28 bioprostheses and 11 mechanical prostheses, thrombosis for 3 bioprostheses and 3 mechanical prostheses, and sudden unexpected death for 7 bioprostheses and 2 mechanical prostheses.

\section{Discussion}

The long-term performances of bioprostheses and mechanical prostheses have had limited documentation to 15 years. Most publications on bioprostheses and mechanical prostheses have dealt with specific valve-related complications: thromboembolism and antithromboembolic-related hemorrhage for mechanical prostheses and limited durability because of structural valve deterioration for bioprostheses. ${ }^{12-14}$ The past 10 to 15 years have seen reduced use of mitral valve replacement in favor of mitral valve reconstruction, especially for degenerative disease.

There have been two large randomized trials comparing the performance of bioprostheses and mechanical prostheses. ${ }^{1-5}$ The United States Veterans Affairs randomized aortic and mitral implantations to the Björk-Shiley spherical disk mechanical prosthesis or the Hancock porcine bioprosthesis. The Veterans Affairs study has produced three publications, with the final report in 2000 documenting their 
15-year experience. ${ }^{1-3}$ The principal long-term findings are as follows: mechanical valves resulted in a lower mortality and lower reoperation rate for aortic valve replacement; the mortality for mitral valve replacement was similar between prosthetic valve types; primary tissue failure after both aortic and mitral valve replacement occurred more frequently with a bioprosthetic valve, especially in patients less than 65 years at implantation; use of the bioprosthetic valve resulted in a lower bleeding rate; and there were no differences between the valve types with regard to other valve-related complications, including thromboembolism and all complications.

The other randomized trial between bioprostheses and a mechanical valve, the Edinburgh trial, was reported on in 1991 and 2003. ${ }^{4,5}$ At 12 years, the study indicated a trend toward better survival with the mechanical valve. The 20year evaluation revealed no difference in survival between the mechanical prosthesis and bioprosthesis populations. The study considered combined death and reoperation as end points and confirmed that patients with mechanical prostheses had improved survival with the original prosthesis intact. This difference became apparent after 8 to 10 years for mitral valve replacement and after 12 to 14 years for aortic valve replacement. Major bleeding was more common with mechanical prostheses, but there was no difference in major embolism or endocarditis. Other studies have identified similar survival trends between bioprostheses and mechanical prostheses. ${ }^{12,13}$ Demirag and investigators ${ }^{14}$ found no difference in survival and reoperation at 10 years in their study evaluating the Biocor porcine bioprosthesis and St Jude Medical mechanical prosthesis in the mitral position. In a 20-year comparison study in an elderly population, bioprostheses did not offer a survival advantage relative to mechanical valves. ${ }^{15}$ That study did determine that anticoagulant-related mortality and morbidity were higher in patients with mechanical valves. Kassai and colleagues ${ }^{16}$ also evaluated survival in a meta-analysis of randomized clinical trials comparing bioprostheses and mechanical prostheses and found no significant influence. Kawachi and coworkers ${ }^{17}$ compared the two prosthesis types in the mitral position and also failed to find any survival benefit. That study also reported for bioprostheses in the mitral position only a small benefit in antithrombogenesis but a prominent disadvantage in altered durability necessitating reoperation.

Jamieson and colleagues ${ }^{18,19}$ have provided considerable documentation supporting the indications for bioprostheses and mechanical prostheses. Bioprostheses are recommended for aortic valve replacement in patients older than 65 years and for mitral valve replacement in patients older than 70 years. ${ }^{6,18,19}$ Mechanical prostheses are the predominant choice of prosthesis type other than for these age groups.
This study assessed the composites of valve-related complications-reoperation, morbidity (permanent impairment), and mortality - by both actuarial and actual methodologies. Jamieson and colleagues ${ }^{6}$ previously reported on valverelated reoperation and valve-related mortality in a porcine bioprosthesis population. The actual freedom for mitral valve replacement at 15 years from valve-related mortality in the age group 61 through 70 years was $79.5 \%$ (actuarial $59.5 \%$ ), and in the age group older than 70 years it was $82.0 \%$ (actuarial 26.1\%). The actual freedom at 15 years from valve-related reoperation in the age group 61 through 70 years was $71.0 \%$ (actuarial $32.6 \%$ ), and in the age group older than 70 years, it was $93.3 \%$ (actuarial $83.4 \%$ ). Those results are not appreciably different from those in this study.

This study compared the performance of bioprostheses and mechanical prostheses to 15 years. Actual freedoms from valve-related reoperation at 15 years for bioprostheses were less than $50 \%$ for patients younger than or equal to 60 years, $75 \%$ for those aged 61 through 70 years, and $92 \%$ for those older than 70 years. Mechanical prostheses had a greater than $94 \%$ actual freedom from valve-related reoperation in all age groups at 12 to 15 years. There was no differentiation between bioprostheses and mechanical prostheses in freedom from valve-related morbidity (permanent neurologic or functional impairment) for all age groups. The freedom from valve-related mortality was extended for all age groups (88\%-99\% at $12-15$ years) for mechanical prostheses, yet relatively stable for all age groups for bioprostheses (81\%-88\% at 15 years). There are no comparable studies in the literature except the previously quoted results for bioprostheses from our institution. ${ }^{6,18}$

This study captured all valve-related morbidity and mortality events for both mechanical prostheses and bioprostheses by censoring patients 31 days after reoperation if the valve type changed and the patients entered into the new valve type group. If the valve type did not change at reoperation, the patients remained in the original groups. This methodology of patient classification precluded the determination of patient survival for mechanical prostheses and bioprostheses, because the patients with successful reoperations were in both arms of the study. The major limitation of the study is that the follow-up of the mechanical prostheses did not afford acceptable numbers of patients at risk between 12 and 15 years.

This comparative evaluation suggests a priority for mechanical prostheses relative to bioprostheses with regard to freedom from valve-related reoperation and valve-related mortality, but not from freedom from valve-related morbidity because of neurologic or functional impairment. The recommendation from this center for mitral valve replacement, as documented in previous publications, was to implant bioprostheses in patients older than 70 years and in selected patients older than 60 years because of life-limiting 
comorbidities. These recommendations remain unchanged after this comparative study. This study, as do our previous studies, provides evidence favoring bioprosthesis use in patients older than 70 years because of the freedom from reoperation. Our recent publication ${ }^{20}$ documented excellent reoperative mortality for structural valve deterioration, with the risk factors for reoperative mortality predominantly emergency status and advanced New York Heart Association functional class IV. An excellent patient surveillance system would avoid high risk reoperative surgery and afford greater freedom from valve-related mortality. The choice of prosthesis for mitral valve replacement goes beyond the consideration of composites of complications that cause reoperation, permanent and functional impairment, or mortality to influential factors inclusive of long-term care with anticoagulation and the risk of nonmorbid thromboembolic events and hemorrhagic events. As stated, the design of this comparative study with crossover of prosthesis type at reoperation to the alternative type precluded the opportunity to evaluate patient survival between bioprostheses and mechanical prostheses.

We acknowledge and appreciate the word processing of this manuscript by Kevin Shillitto.

\section{References}

1. Hammermeister K, Sethi GK, Henderson WG, Grover FL, Oprian C, Rahimtoola SH. Outcomes 15 years after valve replacement with a mechanical versus a bioprosthetic valve: final report of the Veterans Affairs randomized trial. J Am Coll Cardiol. 2000;36:1152-8.

2. Hammermeister KE, Sethi GK, Henderson WG, Oprian C, Kim T, Rahimtoola SH. A comparison of outcomes in men 11 years after heart-valve replacement with a mechanical valve or bioprosthesis. Veterans Affairs Cooperative Study on Valvular Heart Disease. N Engl J Med. 1993;328:1289-96.

3. Hammermeister KE, Henderson WG, Burchfiel CM, Sethi GK, Souchek J, Oprian C, et al. Comparison of outcome after valve replacement with a bioprosthesis versus a mechanical prosthesis: initial 5 year results of a randomized trial. J Am Coll Cardiol. 1987; 10(4):719-32.

4. Bloomfield P, Wheatley DJ,Prescott RJ, Miller HC. Twelve-year comparison of a Björk-Shiley mechanical heart valve with porcine bioprostheses. N Engl J Med. 1991 28;324:573-9.

5. Oxenham H, Bloomfield P, Wheatley DJ, Lee RJ, Cunningham J, Prescott $\mathrm{RJ}$, et al. Twenty-year comparison of the Björk-Shiley mechanical heart valve with porcine bioprostheses. Heart. 2003;89:715-21.

6. Jamieson WRE, Miyagishima RT, Burr LH, Lichtenstein SV, Fradet GJ, Janusz MT. Carpentier-Edwards porcine bioprostheses: clinical performance assessed by actual analysis. J Heart Valve Dis. 2000;9: 530-5.

7. Edmunds LH Jr, Clark RE, Cohn LH, Grunkemeier GL, Miller DC, Weisel RD. Guidelines for reporting morbidity and mortality after cardiac valvular operations. Ad Hoc Liaison Committee for Standardizing Definitions of Prosthetic Heart Valve Morbidity of The American Association for Thoracic Surgery and The Society of Thoracic Surgeons. J Thorac Cardiovasc Surg. 1996;112:708-11.

8. Grunkemeier GL, Li HH, Naftel DC, Starr A, Rahimtoola SH. Longterm performance of heart valve prostheses. Curr Probl Cardiol. 2000;25:73-154.

9. Grunkemeier GL, Starr A, Rahimtoola SH. Clinical performance of prosthetic heart valves. In: Schlant R, Alexander RW, editors. Hurst's the heart. 9th ed. New York: McGraw-Hill; 1998. p.1851-66.
10. Grunkemeier GL, Anderson RP, Miller DC, Starr A. Time-related analysis of nonfatal heart valve complications: cumulative incidence (actual) versus Kaplan-Meier (actuarial). Circulation. 1997;96(9 Suppl):II70-5.

11. Grunkemeier GL, Jamieson WR, Miller DC, Starr A. Actuarial versus actual risk of porcine structural valve deterioration. $J$ Thorac Cardiovasc Surg. 1994;108:709-18.

12. Myken PS, Caidahl K, Larsson P, Larsson S, Wallentin I, Berggren HE. Mechanical versus biological valve prosthesis: a ten-year comparison regarding function and quality of life. Ann Thorac Surg. 1995;60(2 Suppl):S447-52.

13. Kobayashi Y, Eishi K, Nagata S, Nakano K, Sasako Y, Kobayashi J, et al. Choice of replacement valve in the elderly. J Heart Valve Dis. 1997;6:404-9.

14. Demirag M, Kirali K, Omeroglu SN, Mansuroglu D, Akinci E, Ipek G, et al. Mechanical versus biological valve prosthesis in the mitral position: a 10-year follow up of St Jude Medical and Biocor valves. J Heart Valve Dis. 2001;10:78-83.

15. Sidhu P, O'Kane H, Ali N, Gladstone DJ, Sarsam MA, Campalani G, et al. Mechanical or bioprosthetic valves in the elderly: a 20-year comparison. Ann Thorac Surg. 2001;71(5 Suppl):S257-60.

16. Kassai B, Gueyffier F, Cucherat M, Boissel JP. Comparison of bioprosthesis and mechanical valves, a meta-analysis of randomised clinical trials. Cardiovasc Surg. 2000;8:477-83.

17. Kawachi Y, Tokunaga K. Clinical comparative study between mitral mechanical and bioprosthetic valves - what is the benefit of bioprosthetic valves in the mitral position? Jpn Circ J. 1990;54:1525-34.

18. Jamieson WRE, Burr LH, Miyagishima RT, Germann E, Anderson WN. Actuarial versus actual freedom from structural valve deterioration with the Carpentier-Edwards porcine bioprostheses. Can J Cardiol. 1999;15:973-8.

19. Jamieson WRE, Tyers GF, Janusz MT, Miyagishima RT, Munro AI, Ling $\mathrm{H}$, et al. Age as a determinant for selection of porcine bioprostheses for cardiac valve replacement: experience with CarpentierEdwards standard bioprosthesis. Can J Cardiol. 1991;7:181-8.

20. Jamieson WRE, Burr LH, Miyagishima RT, Janusz MT, Fradet GJ, Lichtenstein SV, et al. Reoperation for bioprosthetic mitral structural failure: risk assessment. Circulation. 2003;108(10 Suppl):II98-102.

\section{Discussion}

Dr Colleen Sintek (Los Angeles, Calif). Congratulations on your clear presentation of a comparative analysis of a large series of mitral valve replacements. You, along with Gary Grunkemeier, Al Starr, and Craig Miller, have previously demonstrated that the usual actuarial curves do not provide meaningful percentages of structural valve deterioration or valve-related morbidity. The actuarial event-free curve for a nonfatal event estimates the eventfree probability for a population in which death has been eliminated. This overestimates the percentages for valve-related reoperation and valve-related morbidity, because many patients die before these events occur. Of more direct clinical relevance, as you have stated, is the percentage of patients who have valve-related complications before death.

Your study proposes that valve-related reoperation favors mechanical prostheses for all but patients older than 70 years, that valve-related mortality favors mechanical relative to bioprosthetic valves for patients 70 years of age and younger, and that valverelated morbidity is the same for mechanical and bioprosthetic valves in all age groups. You conclude that patients 70 years of age and younger requiring mitral valve replacement should receive a mechanical valve and that for those older than 70 there is no advantage of bioprostheses relative to mechanical prostheses.

I have several concerns and questions about this report. Your definition of valve-related morbidity excluded nonpermanent events. You have already elaborated on that to some extent, but I 
did note that in a previous study of your own mitral mechanical replacements you reported a thromboembolic event rate of 5.5 events/100 patient-years and a hemorrhagic complication rate of 1.5 events/100 patient-years. That is opposed to the 0.57 events/ 100 patient-years valve-related morbidity that you report in this report. Did you exclude peripheral emboli that were successfully treated surgically or hemorrhage that required transfusion or a thrombosed valve that was successfully treated with thrombolytic therapy? Do you believe that such events should be considered when selecting a prosthesis for a given patient?

Dr Jamieson. You are right, Dr Sintek. In that group of patients, we did have an incidence of thromboembolism of 5.0 events/100 patient-years, and that included minor events, major events, RIND (reversible ischemic neurologic deficits), and thrombosis. For this particular study, we went on the understanding that the patients were really concerned whether they would die from the valve, would have a reoperation, or would have permanent impairment. We did alter that definition, and we do describe what we did here. There is no question that the nonmorbid events are still important to the patient, but we did try and differentiate here as to whether or not the patient had a permanent neurologic impairment in particular.

Dr Sintek. I am concerned about the shorter follow-up for your mechanical valve population. In fact, I am not sure that there are enough patients older than 70 years at risk-even at 10 years, there were only 7 patients - to come to a valid conclusion in comparison with the bioprosthetic group.

Dr Jamieson. Yes, I do agree with you. We commenced this follow-up about 3 years ago, and we are concerned about the lesser number of patients at risk at the advanced years in the mechanical prosthesis population. It is conceivable even today that if we went back and followed those mechanical valves again, we would add greater numbers at the extended years. I think that is part of this whole work in process. That is important, and I take your criticism as valid.

Dr Sintek. You found no difference in the need for reoperation in patients undergoing concomitant $\mathrm{CABG}$ with mitral valve replacement, although previous CABG was a predictor for valverelated reoperation. Could you elaborate on this?

Dr Jamieson. I'll answer the second question first. Those patients who had previous CABG obviously have an anticipated reduced life expectancy because of this comorbid disease. Consequently, because of reduced life expectancy, they have a reduced potential for reoperation. The other important part of this though, is that among those patients for whom we did concomitant CABG at the time of reoperation, that was not a factor. This is probably related to a study we presented at the American Heart Association meeting in November, in which we found that in the last 7 years we reduced our valve-related mitral mortality to $3.4 \%$, among those with concomitant coronary bypass, to $2.8 \%$, so these are influential factors in here that are coming through. Concomitant coronary bypass is not a predictor because of our last 8-year experience.

Dr Sintek. This is a comparative study, not randomized. Did cardiac rhythm play a role in selecting a prosthesis for a given patient? And could you share with us your anticoagulation protocol for both bioprosthetic and mechanical valves in the mitral position?

Dr Jamieson. We looked at rhythm as a factor here, and it did not come out in univariate analysis as being a predictor. As far as anticoagulation goes, the majority (and I just say the majority because there are 15 surgeons in this group) do use anticoagulation early and for at least 6 weeks in those patients who are in sinus rhythm and do not have other extenuating factors as risk factors for venous stasis in the atrium, and those patients would be anticoagulated and then switched to aspirin if they had a bioprosthesis and obviously continued on if the prosthesis was mechanical. The patients with mechanical prostheses were always anticoagulated early. In recent years, we have also been using low-dose heparin, and I might share with you some information from the AVERT (Artificial Valve Endocarditis Reduction Trial) trial that patients who were on heparin early postoperatively also had a reduced incidence of late thromboembolic events. This is a factor not yet fully explored.

Dr Sintek. Last, applying the lessons learned from your study, what type valve would you implant in a 69-year-old man in sinus rhythm requiring mitral valve replacement and CABG?

Dr Jamieson. Well, there are certainly factors here that are not readily apparent. You have to look at the bioprosthesis that you are using. A large group of these patients were implanted with Carpentier-Edwards supra-annular valves in the mitral position, and we found a few years ago and actually presented at the Western Thoracic Surgical Association meeting that the supra-annular porcine valve did not fare as well as the Carpentier-Edwards PERIMOUNT pericardial valve. We have now a 6-year experience with the Medtronic Mosaic. The other factor to be considered, coming out of the experience in Sweden, and this perhaps is going to be brought out next weekend at the Society for Heart Valve Disease, is that those porcine valves that are formulated as a tricomposite configuration with three separate leaflets appear to have extended durability in the mitral position. So looking at those things and what is coming forward in the future and what will be available even in North America on a trial basis within a couple of months, I might certainly consider an advanced generation bioprosthesis for the patient in sinus rhythm undergoing concomitant CABG. 